\title{
Message-Splitting for Interference Cancellation in Multibeam Satellite Systems
}

\author{
Tomás Ramírez*, Carlos Mosquera*, Màrius Caus ${ }^{\dagger}$, Adriano Pastore ${ }^{\dagger}$, Monica Navarro ${ }^{\dagger}$, Nele Noels ${ }^{\ddagger}$ \\ *atlanTTic research center, University of Vigo, Galicia, Spain. \\ ${ }^{\dagger}$ Centre Tecnològic de Telecomunicacions de Catalunya (CTTC), Castelldefels, Barcelona, Spain

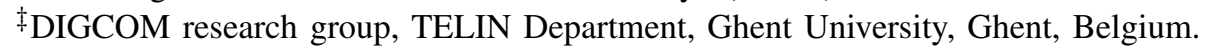

\begin{abstract}
This paper investigates a novel technique to deal with the interference in the forward link of multibeam satellite systems when aggressive frequency reuse schemes are employed. Taking into account only magnitude information about the forward channel, the gateway judiciously splits the messages to be transmitted into private and public parts. At the receive terminals, partial cancellation of the public messages is applied prior to private message detection. The practical significance of the absence of channel phase information is stressed and complemented by some additional insights on the implementation. Our numerical results show that, in terms of average total throughput, this technique combined with a 2-colour frequency reuse scheme can outperform a classic orthogonal system with a conservative 4-colour frequency reuse scheme, despite the additional co-channel interference.
\end{abstract}

\section{INTRODUCTION}

Traditionally, multibeam satellite communication systems have been designed to avoid interference among beams. In most of the cases, 4-colour frequency reuse schemes are able to provide the required isolation between co-channel beams. In this case, the residual interference can be treated as noise without a significant system performance degradation. The beauty of this approach is that beams can be independently processed, which simplifies several operations such as the selection of users to be served and the rate selection. However, the need to increase the satellite throughput calls for the adoption of more aggressive frequency reuse schemes. It is important to remark that this approach comes at the expense of an increased co-channel interference, which may have a magnitude similar to that of the desired signal. Hence, it is of paramount importance to endow the system with interference mitigation capabilities.

To unleash the full potential of full frequency reuse schemes in the multibeam satellite context, it is crucial to employ centralised pre-coding techniques [1]-[3]. The use of linear pre-coding is of special interest, because matrix algebra tools can be harnessed to efficiently generate the precoded signal. It is worth mentioning that the implementation of pre-coding demands for full channel state information at the transmitter (CSIT), i.e. amplitude and phase of the channel estimates, which has an impact on the return signalling.

The practical implementation aspects of pre-coding have motivated the investigation of interference mitigation techniques that require only partial CSIT, e.g. the signal-tonoise ratio (SNR) of the links. Pursuing this idea, some publications have shown throughput gains by placing the complexity at the receive side [4]-[8]. All these strategies rely on advanced receivers that are able to decode several non-orthogonal signals. Usually, to keep the complexity at a reasonable level, decoding strategies only process two signals. For this reason, de-centralised interference mitigation techniques are mostly applied in 2-colour frequency reuse schemes using time division multiplexing to serve 1 user per beam in each time slot. In this scenario there is at most one strong interference signal. So, if users and their corresponding spot beams are judiciously grouped in pairs, the interference outside the cluster will be minimised. Interestingly, the system model of each pair or cluster lies within the broadcast channel framework, allowing us to benefit from theory developed for this class of channels. In this regard, the authors in [9] have investigated two schemes referred to as superposition coding with successive cancellation decoding (SC-SCD) and non-coherent rate splitting (NCRS). Both schemes in [9] are based on the non-orthogonal superposition of signals which are successively decoded at the receivers. We focus here on NCRS, which as opposed to SC-SCD, does not need high SNR imbalances between users for satisfactory performance. Please note that in multi-beam satellite settings, the SNR imbalance accountable to the location of static users does not usually exceed 3-4 dB.

In this work a rate-splitting technique is presented for multibeam satellite systems to cope with the interference when aggressive frequency reuse schemes are employed. Focusing on the practical implementation, low complexity solutions are devised.

The rest of the paper is organized as follows. Section II defines the satellite system model. Next, the theoretical framework is shown in Section III. NCRS is presented in Section IV. The optimization of NCRS is pursued in Section $\mathrm{V}$, and after that the system simulation results are shown in Section VI. Finally, some conclusions are given in Section VII.

Notation: Upper (lower) boldface letters denote matrices (vectors). (. $)^{H}, \mathbf{I}_{N}$ denote Hermitian transpose and $N \times N$ identity matrix, respectively. $\mathbb{E}[\cdot]$ is the expected value operator.

\section{SYSTEM MODEL}

In this work we focus on the forward link of a multibeam satellite communication system. The satellite is equipped with an array fed reflector antenna that is able to generate $K$ spotbeam areas. Assuming perfect synchronization, an ideal feeder link and neglecting non-linear effects in the satellite 
payload, the values received by $K$ users at a given time instant can be written as

$$
\mathbf{y}=\mathbf{H} \mathbf{x}+\mathbf{w}
$$

where $\mathbf{y} \in \mathbb{C}^{K \times 1}$ is the vector of received signals given by $\mathbf{y}=\left[\begin{array}{llll}y_{1}[k] & y_{2}[k] & \ldots & y_{K}[k]\end{array}\right]^{T}, \mathbf{H} \in \mathbb{C}^{K \times K}$ is the channel matrix, $\mathbf{x} \in \mathbb{C}^{K \times 1}$ is the vector of transmitted signals given

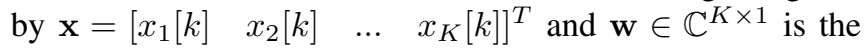
noise vector given by $\mathbf{w}=\left[\begin{array}{llll}w_{1}[k] & w_{2}[k] & \ldots & w_{K}[k]\end{array}\right]^{T}$. To keep the notation simple, the time index is dropped from now on. The global communication system lies within the Multiple Input Single Output Broadcast Channel (MISO BC) framework for the case of $K$ single antenna user terminals, one per beam, which are served by $K$ antenna feeds. All the antenna feeds transmit with the same power, so that $\mathbb{E}\left[\mathbf{x x}^{\mathrm{H}}\right]=\frac{P}{K} \mathbf{I}_{K}$ for a total power $P$. The Additive White Gaussian Noise (AWGN) $\mathbf{w}$ is such that $\mathbb{E}\left[\mathbf{w w}^{\mathrm{H}}\right]=\sigma^{2} \mathbf{I}_{K}$.

The $(q, m)$ th entry of the matrix $\mathbf{H}$ is given by:

$$
h_{q m}=e^{j \phi_{q m}} \frac{\sqrt{G_{R} G_{q m}}}{\sqrt{A_{q}} 4 \pi d_{q} / \lambda} .
$$

Here, the term $G_{R}$ refers to the receive antenna gain and $G_{q m}$ represents the transmit antenna gain from beam $m$ to the $q$-th user terminal. As for the rest of the terms, $\lambda$ is the carrier wavelength, $\phi_{q m}$ is the phase rotation introduced by the channel and $d_{q}$ is the distance from the satellite to the $q$-th user.

In order to keep the complexity at reasonable levels, the beams using the same frequency colour are grouped into pairs $(q, m)$, whereby the corresponding users $q$ and $m$ only process two signals, namely, $x_{q}$ and $x_{m}$, treating all the background interference from outside the $(q, m)$ beam pair as noise. For a given beam pair, without loss of generality denoted as $(1,2)$, the signals received at users 1 and 2 can be written as

$$
\begin{aligned}
& y_{1}=h_{11} x_{1}+h_{12} x_{2}+z_{12}, \\
& y_{2}=h_{21} x_{1}+h_{22} x_{2}+z_{21},
\end{aligned}
$$

where $z_{12}$ and $z_{21}$ are equivalent noise samples with

$$
z_{i j}=w_{i}+\sum_{p=3}^{M} h_{i p} x_{p}, \quad i=1,2
$$

where $M \leq K$ is the number of beams using the same frequency colour as the beam pair $(1,2)$. Assuming Gaussian code books, the $z_{i j}$ are zero-mean Gaussian random variables with variance

$$
\sigma_{i j}^{2}=\sigma^{2}+I_{i j}
$$

where

$$
I_{i j}=\sum_{p=3}^{M} \frac{P}{K}\left|h_{i p}\right|^{2}, \quad i=1,2 .
$$

The receivers are assumed to operate with full knowledge of $h_{i j}$, whereas the gateway will be assumed to have access to channel magnitude information $\left|h_{i j}\right|$ only. When it comes to reporting the channel state information from the user to the gateway, the limited signal overhead in the uplink and the long delay times are the main impairments of the CSIT.
If only the channel magnitudes have to be reported, the required amount of feedback reduces significantly; moreover, assuming slow fading, the magnitude values are practically constant during the time-delay window. Hence, as compared to the case of full CSIT, the CSIT uncertainty is practically removed at the cost of working with less CSIT (magnitudes only).

Once a pair $(q, m)$ is formed, the link between the pair $(q, m)$ and the $q$ th receiver is characterized by the following metrics:

$$
\gamma_{q n}=\frac{\frac{P}{K}\left|h_{q n}\right|^{2}}{\sigma^{2}+I_{q m}}
$$

where $n \in\{q, m\}$. By swapping the roles of $q$ and $m$, we obtain the corresponding metrics $\gamma_{m n}$ at the $m$ th receiver.

\section{THEORETICAL FRAMEWORK}

The Degrees of Freedom (DoF) metric is an asymptotic characterization of Multiple-Input Multiple-Output (MIMO) channels, and can be interpreted as the total number of interference-free streams that can be simultaneously supported in a single channel use. The DoF metric is defined as

$$
\mathrm{DoF}=\lim _{P \rightarrow \infty} \frac{C(P)}{\log _{2} P}
$$

with $C(P)$ the sum capacity for the total transmit power $P$ [10].

With full CSIT, the DoF of the MISO BC are $K$, and can be achieved by means of the Zero Forcing (ZF) precoder [11]. With partial CSIT, [12] and [13] have obtained independently the DoF if the channel estimation error scales as $\sim$ SNR $^{-\beta}$. The $K$ user MISO BC has $1+(K-1) \beta$ DoF. For perfect CSIT $(\beta=1)$, the DoF are $K$, whereas for finite precision CSIT $(\beta=0)$, the DoF collapse to 1 . In [12], a rate splitting approach, akin to the one employed in the Han-Kobayashi scheme [14], is used for construction of the transmit codes, whereas [13] uses an Aligned Image Sets (AIS) argument.

One of the main limitations of the DoF framework is that it treats all non-zero channels as equally strong in the high SNR limit, ignoring the strength of the different signals, which are mapped into a DoF each. Thus, the DoF metric does not provide much insight on how to manage interference effectively when some signals are significantly stronger or weaker than others [10]. The generalized degrees of freedom (GDoF) metric, first introduced in [15], and more recently exploited in [16], opens up the scope of DoF by differentiating between weak and strong interference regimes. The GDoF framework serves to approximate the interference-limited performance in the high-SNR regime. Under this framework, the main result in [16] advocates interference enhancement as a way to increase the GDoF above one under finite precision CSIT, i.e, the transmitters are aware of the coarse channel strength parameters. From now on, we label as interference enhancement (ENH), the technique based on this idea of interference reinforcement. The ENH technique tries to reinforce part of the interfering 


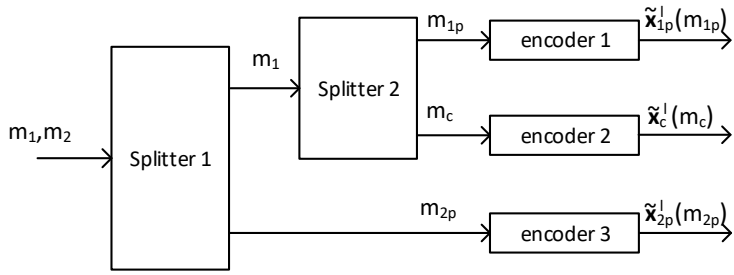

Figure 1. Interference Enhancement (ENH) encoding process.

term in one of the receiver terminals so it can be decoded and removed. Following the ENH encoding scheme which is shown in Figure 1 for $K=2$ users, the transmitted ENH signals are given by

$$
\begin{aligned}
& x_{1}=\sqrt{\frac{P}{2}\left(1-\lambda_{1}\right)} \tilde{x}_{c}+\sqrt{\frac{P}{2} \lambda_{1}} \tilde{x}_{1_{p}}, \\
& x_{2}=\sqrt{\frac{P}{2}\left(1-\lambda_{2}\right)} \tilde{x}_{c}+\sqrt{\frac{P}{2} \lambda_{2}} \tilde{x}_{2_{p}},
\end{aligned}
$$

where $\tilde{x}_{c}, \tilde{x}_{1_{p}}$ and $\tilde{x}_{2_{p}}$ are the symbols of the length- $l$ codewords $\tilde{\mathbf{x}}_{c}^{l}$, $\tilde{\mathbf{x}}_{1_{p}}^{l}$ and $\tilde{\mathbf{x}}_{2_{p}}^{l}$ at a given time instant. The encoding process goes as follows. One of the messages, without loss of generality the first user's message, must be split as $m_{1}=\left(m_{c}, m_{1_{p}}\right)$. The user 2's message is given as $m_{2}=m_{2_{p}}$. These messages $m_{1_{p}}, m_{c}$ and $m_{2_{p}}$ are encoded into $\tilde{\mathbf{x}}_{c}^{l}\left(m_{c}\right), \tilde{\mathbf{x}}_{1_{p}}^{l}\left(m_{1_{p}}\right), \tilde{\mathbf{x}}_{2_{p}}^{l}\left(m_{2_{p}}\right)$ respectively. The $m_{1_{p}}$ and $m_{2_{p}}$ messages are private messages but to be decoded only by user 1 and user 2 , respectively, while $m_{c}$ acts as a public message intended to user 1 to be decodable by both users. At the receiver side, receiver 1 first decodes the message $m_{c}$, treating everything else as noise. After reconstructing $\tilde{\mathbf{x}}_{c}^{l}$ and subtracting its contribution from the received signal, receiver 1 further decodes the codeword $\tilde{\mathbf{x}}_{1_{p}}^{l}$ for its desired message $m_{1_{p}}$. Receiver 2 proceeds similarly, its desired message $m_{2_{p}}$ is recovered after decoding, subtracting and discarding the public message $\tilde{\mathbf{x}}_{c}^{l}$.

\section{Non-Coherent Rate-Splitting}

The practical performance of the ENH encoding scheme depends on the specific channel values. The relative phase offset between the channel coefficients $h_{11}$ and $h_{12}\left(h_{21}\right.$ and $h_{22}$ ) will determine the magnitude of the received common message at receiver 1 (receiver 2). For example, if we substitute the ENH signals from (10) in (3), the contribution from the common message signal at the receiver 1 is

$\tilde{x}_{c}\left[\sqrt{\left(1-\lambda_{1}\right)}\left|h_{11}\right|+\sqrt{\left(1-\lambda_{2}\right)}\left|h_{12}\right| e^{j\left(\phi_{11}-\phi_{12}\right)}\right] e^{j \phi_{12}}$

The relative phase offset $\phi_{11}-\phi_{12}$, which is unknown to the transmitter, will determine the maximum admissible rate. In order to overcome this limitation, Non-Coherent RateSplitting (NCRS) is presented as a way to avoid the channel phase dependence and, consequently, to reduce the level of required CSIT. Similarly to ENH, one of the messages is split into a public and a private message, and then the public message $m_{c}$ is split again into $m_{1_{c}}$ and $m_{2_{c}}$ which

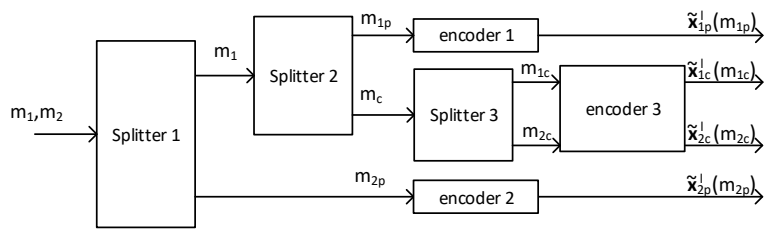

Figure 2. Non-Coherent Rate Splitting (NCRS) scheme.

are encoded into $\tilde{\mathbf{x}}_{2_{c}}^{l}\left(m_{1_{c}}\right)$ and $\tilde{\mathbf{x}}_{2_{c}}^{l}\left(m_{2_{c}}\right)$, respectively. A particular instance of the NCRS encoding scheme is shown in Figure 2. More generally, the public message can be generated by combining information from both users. In contrast to ENH, the NCRS codeword corresponding to the public message is now given by $\mathbf{x}_{c}^{l}=\left[\begin{array}{ll}\tilde{x}_{1_{c}}^{l} & \tilde{\mathbf{x}}_{2_{c}}^{l}\end{array}\right]$ instead of a single codeword $\tilde{\mathbf{x}}_{c}^{l}$. The transmitted NCRS signals, read as

$$
\begin{aligned}
& x_{1}=\sqrt{\frac{P}{2}\left(1-\lambda_{1}\right)} \tilde{x}_{1_{c}}+\sqrt{\frac{P}{2} \lambda_{1}} \tilde{x}_{1_{p}}, \\
& x_{2}=\sqrt{\frac{P}{2}\left(1-\lambda_{2}\right)} \tilde{x}_{2_{c}}+\sqrt{\frac{P}{2} \lambda_{2}} \tilde{x}_{2_{p}},
\end{aligned}
$$

where $\mathbb{E}\left[\begin{array}{ll}\left|x_{1}\right|^{2} & x_{1} x_{2}^{*} \\ x_{2} x_{1}^{*} & \left|x_{2}\right|^{2}\end{array}\right]=\frac{P}{2} \boldsymbol{I}$ and $\tilde{x}_{1_{c}}, \tilde{x}_{2_{c}}, \tilde{x}_{1_{p}}$ and $\tilde{x}_{2_{p}}$ are the symbols of the length- $l$ codewords $\tilde{\mathbf{x}}_{1_{c}}^{l}, \tilde{\mathbf{x}}_{2_{c}}^{l}, \tilde{\mathbf{x}}_{1_{p}}^{l}$ and $\tilde{\mathbf{x}}_{2_{p}}^{l}$ at a given time instant, respectively.

In the same way as $\mathrm{ENH}$, the receivers first decode the codeword $\mathrm{x}_{c}^{l}$ of the public message, and then recover their respective private message. The NCRS rate regions $\mathcal{R}_{1}$ and $\mathcal{R}_{2}$, which are obtained when the public message is assigned to user 1 and to user 2 respectively, are given by

$$
\begin{aligned}
& \mathcal{R}_{1}=\left\{R_{1}<I\left(Y_{1}, \tilde{X}_{1_{p}} \mid X_{c}\right)+\min \left\{I\left(Y_{1}, X_{c}\right), I\left(Y_{2}, X_{c}\right)\right\},\right. \\
& \left.R_{2}<I\left(Y_{2}, \tilde{X}_{2_{p}} \mid X_{c}\right)\right\} \\
& \mathcal{R}_{2}=\left\{R_{1}<I\left(Y_{1}, \tilde{X}_{1_{p}} \mid X_{c}\right)\right. \\
& \left.R_{2}<I\left(Y_{2}, \tilde{X}_{2_{p}} \mid \tilde{X}_{c}\right)+\min \left\{I\left(Y_{1}, \tilde{X}_{c}\right), I\left(Y_{2}, X_{c}\right)\right\}\right\},
\end{aligned}
$$

where $I(X ; Y)$ denotes the mutual information between random variables $X$ and $Y$. The achievable rate region is given by the convex hull of the union $\mathcal{R}_{1} \cup \mathcal{R}_{2}$. The corresponding expressions for Gaussian codebooks and the channel parametrized by (8), are given by (16) and (17).

\section{RATE OPTIMIZATION}

The global optimization of NCRS requires to address jointly the beam pairing and associated rate selection. The high complexity of this process makes it more practical to decouple both problems, so that a heuristic algorithm to pair beams is employed. After the user positions are acquired, beams are paired so that $\gamma_{12}$ and $\gamma_{21}$ are as high as possible, 


$$
\begin{aligned}
\mathcal{R}_{1}= & \left\{R_{1}<\log _{2}\left(1+\frac{\lambda_{1} \gamma_{11}}{1+\lambda_{2} \gamma_{12}}\right)+\min \left\{\log _{2}\left(1+\frac{\left(1-\lambda_{1}\right) \gamma_{11}+\left(1-\lambda_{2}\right) \gamma_{12}}{1+\lambda_{1} \gamma_{11}+\lambda_{2} \gamma_{12}}\right), \log _{2}\left(1+\frac{\left(1-\lambda_{1}\right) \gamma_{21}+\left(1-\lambda_{2}\right) \gamma_{22}}{1+\lambda_{1} \gamma_{21}+\lambda_{2} \gamma_{22}}\right)\right\}\right. \\
& \left.R_{2}<\log _{2}\left(1+\frac{\lambda_{2} \gamma_{22}}{1+\lambda_{1} \gamma_{21}}\right)\right\} \\
\mathcal{R}_{2}= & \left\{R_{1}<\log _{2}\left(1+\frac{\lambda_{1} \gamma_{11}}{1+\lambda_{2} \gamma_{12}}\right)\right. \\
& \left.R_{2}<\log _{2}\left(1+\frac{\lambda_{2} \gamma_{22}}{1+\lambda_{1} \gamma_{21}}\right)+\min \left\{\log _{2}\left(1+\frac{\left(1-\lambda_{1}\right) \gamma_{11}+\left(1-\lambda_{2}\right) \gamma_{12}}{1+\lambda_{1} \gamma_{11}+\lambda_{2} \gamma_{12}}\right), \log _{2}\left(1+\frac{\left(1-\lambda_{1}\right) \gamma_{21}+\left(1-\lambda_{2}\right) \gamma_{22}}{1+\lambda_{1} \gamma_{21}+\lambda_{2} \gamma_{22}}\right)\right\}\right\}
\end{aligned}
$$

that is beams are paired with the most interfering beam. Once pairs are formed, the parameters $\lambda_{1}$ and $\lambda_{2}$ in Section IV are obtained for sum-rate optimization.

\section{A. Beam pairing}

The selection of the best pairs of beams is a problem of combinatorial complexity, so that a heuristic approach has been taken to pair users for NCRS implementation. In an effort to address the most significant interfering beam, while minimizing the background interference posed by the rest of the beams, we try to maximize $\left|h_{q m}\right|^{2} / I_{q m}$ in (8). The heuristic algorithm to implement this follows a greedy approach, such that the pairs with lower $I_{q m}$ are chosen first.

\section{B. Rate selection}

The optimization of the sum-rate $R_{1}+R_{2}$ departs from the rate regions in (16) and (17). The sum rate is identical in both cases, since the final destination of the public message is irrelevant in terms of aggregated rates. The maximum achievable rate of the public message $R_{c}$ is given by $\min \left(R_{1_{c}}, R_{2_{c}}\right)$, with

$$
\begin{aligned}
& R_{1_{c}}=\log _{2}\left(\frac{1+\gamma_{11}+\gamma_{12}}{1+\lambda_{1} \gamma_{11}+\lambda_{2} \gamma_{12}}\right) \\
& R_{2_{c}}=\log _{2}\left(\frac{1+\gamma_{21}+\gamma_{22}}{1+\lambda_{1} \gamma_{21}+\lambda_{2} \gamma_{22}}\right) .
\end{aligned}
$$

The optimization of $\lambda_{1}$ and $\lambda_{2}$ needs to be addressed for both cases $R_{1_{c}} \leq R_{2_{c}}$ and $R_{1_{c}} \geq R_{2_{c}}$, each set by the most restrictive terminal:

$$
\begin{aligned}
& R_{1_{c}} \leq R_{2_{c}}: a+b \lambda_{1}+c \lambda_{2} \geq 0 \\
& R_{1_{c}} \geq R_{2_{c}}: a+b \lambda_{1}+c \lambda_{2} \leq 0
\end{aligned}
$$

with

$$
\begin{aligned}
& a=\frac{1}{\alpha}-\frac{1}{\beta} \\
& b=\frac{\gamma_{11}}{\alpha}-\frac{\gamma_{21}}{\beta} \\
& c=\frac{\gamma_{12}}{\alpha}-\frac{\gamma_{22}}{\beta}
\end{aligned}
$$

and

$$
\begin{gathered}
\alpha=1+\gamma_{11}+\gamma_{12}, \\
\beta=1+\gamma_{21}+\gamma_{22} .
\end{gathered}
$$

The two optimization problems which need to be solved are

$$
\begin{array}{cl}
\underset{0 \leq \lambda_{1}, \lambda_{2} \leq 1}{\operatorname{argmax}} & R_{1_{p}}+R_{2_{p}}+R_{1_{c}} \\
\text { s.t. } & a+b \lambda_{1}+c \lambda_{2} \geq 0 \\
& R_{1_{p}}+R_{2_{p}}+R_{2_{c}} \\
\underset{0 \leq \lambda_{1}, \lambda_{2} \leq 1}{\operatorname{argmax}} & a+b \lambda_{1}+c \lambda_{2} \leq 0 \\
\text { s.t. } & a+
\end{array}
$$

which are respectively equivalent to

$$
\begin{array}{cl}
\underset{0 \leq \lambda_{1}, \lambda_{2} \leq 1}{\operatorname{argmax}} & \log _{2}\left(\left(1+\gamma_{11}+\gamma_{12}\right) \frac{1+\lambda_{1} \gamma_{21}+\lambda_{2} \gamma_{22}}{\left(1+\lambda_{1} \gamma_{21}\right)\left(1+\lambda_{1} \gamma_{12}\right)}\right) \\
\text { s.t. } & a+b \lambda_{1}+c \lambda_{2} \geq 0
\end{array}
$$

$$
\begin{array}{cl}
\underset{0 \leq \lambda_{1}, \lambda_{2} \leq 1}{\operatorname{argmax}} & \log _{2}\left(\left(1+\gamma_{21}+\gamma_{22}\right) \frac{1+\lambda_{1} \gamma_{11}+\lambda_{2} \gamma_{12}}{\left(1+\lambda_{1} \gamma_{21}\right)\left(1+\lambda_{1} \gamma_{12}\right)}\right) \\
\text { s.t. } & a+b \lambda_{1}+c \lambda_{2} \leq 0
\end{array}
$$

The maximization can be pursued by applying a sequential quadratic programming method [17].

\section{IMPLEMENTATION ASPECTS}

This section aims at providing insights on the implementation and the complexity of practical decoders for the proposed NCRS scheme.

All throughout the preceding analysis, we have assumed that the four transmitted symbol sequences $\tilde{x}_{1_{c}}[k], \tilde{x}_{2_{c}}[k]$, $\tilde{x}_{1_{p}}[k]$ and $\tilde{x}_{2_{p}}[k], k=1,2, \ldots$, consist of independent Gaussian random variables. In practice, however, discrete modulation and coding schemes (MODCODs) will be employed to generate four symbol sequences $\tilde{\boldsymbol{x}}_{1_{c}}, \tilde{\boldsymbol{x}}_{2_{c}}, \tilde{\boldsymbol{x}}_{1_{p}}$ and $\tilde{\boldsymbol{x}}_{2_{p}}$, where $\tilde{\boldsymbol{x}}_{i_{j}}$ denotes a vector with components $\tilde{x}_{i_{j}}[k]$. In the following, we assume the adoption of multilevel coding where the messages are split into four bit streams $\boldsymbol{b}_{1_{c}}, \boldsymbol{b}_{2_{c}}$, $\boldsymbol{b}_{1_{p}}$ and $\boldsymbol{b}_{2_{p}}$, and each stream $\boldsymbol{b}_{i_{j}}=\left(b_{i_{j}}[1], b_{i_{j}}[2], \ldots\right)$ is encoded by an off-the-shelf MODCOD. Specifically, it is assumed that each bit stream is first encoded by a turbolike binary channel code and that the coded bits are then interleaved and subsequently mapped to a symbol constellation. In the following, we derive receivers for detecting the useful bit streams in the received composite signal. As will be shown, the structure of these receivers naturally casts into the general framework of factor graphs (FG) and the sumproduct algorithm (SPA) [18].

Let $\boldsymbol{y}_{q}$ be an observation vector that groups the relevant samples received by user $q$. It is well known that a maximum 
a posteriori (MAP) bit-by-bit detector, which simultaneously maximizes the marginal bit a posteriori probability (APP) $p\left(b_{i_{j}}[k] \mid \boldsymbol{y}_{q}\right)$ of every bit from every MODCOD encoder, is optimum in the sense that it minimises the bit error probability. Unfortunately, the exact computation of the quantities $p\left(b_{i_{j}}[k] \mid \boldsymbol{y}_{q}\right)$ is often too complex as it involves marginalizing the joint bit APP $p\left(\boldsymbol{b} \mid \boldsymbol{y}_{q}\right)$, with $\boldsymbol{b}=\left(\boldsymbol{b}_{1_{c}}, \boldsymbol{b}_{2_{c}}, \boldsymbol{b}_{1_{p}}, \boldsymbol{b}_{2_{p}}\right)$. Therefore, it is common practice to replace the exact bit APPs by approximations that can be computed more efficiently by applying an instance of the SPA to a FG that represents a suitable factorization of the joint APP $p\left(\boldsymbol{b}, \tilde{\boldsymbol{x}} \mid \boldsymbol{y}_{q}\right)$ with $\tilde{\boldsymbol{x}}=\left(\tilde{\boldsymbol{x}}_{1_{c}}, \tilde{\boldsymbol{x}}_{2_{c}}, \tilde{\boldsymbol{x}}_{1_{p}}, \tilde{\boldsymbol{x}}_{2_{p}}\right)$.

An obvious FG is shown in Figure 3 . It consists of 4 nodes ( $\left.f_{\text {MODCOD }, i}, i=1,2,3,4\right)$ that impose the individual MODCOD constraints to the variable pairs $\left(\boldsymbol{b}_{i_{j}}, \tilde{\boldsymbol{x}}_{i_{j}}\right)$ for $i=1,2$ and $j=p, c$, and a large number of nodes ( 1 per component $k$ of $\boldsymbol{y}_{q}$, denoted as F) that impose the observation constraints to the variable quadruplets $\left(\tilde{x}_{1_{c}}[k], \tilde{x}_{2_{c}}[k], \tilde{x}_{1_{p}}[k], \tilde{x}_{2_{p}}[k]\right)$. Applying the SPA to this FG results in an iterative receiver in which 4 independently operating decoders repeatedly exchange extrinsic information with a multi-user symbol detector (MUD). As compared to orthogonal signalling, the resulting receiver is much more complex. Not only does it involve 4 decoders (instead of only 1) and an additional MUD, it also requires that each of these units is run repeatedly. The major part of the added complexity stems from the fact the SPA involves iterations between the different MODCOD decoders. At each iteration, the decoder inputs require updating. It can be shown that for the $i_{j}$ th decoder this involves a number of operations proportional to $\prod_{k_{l} \neq i_{i}} M^{\left(k_{l}\right)}$, with $M^{\left(k_{l}\right)}$ the constellation size of the MODCOD employed for the $k_{l}$ th bit stream.

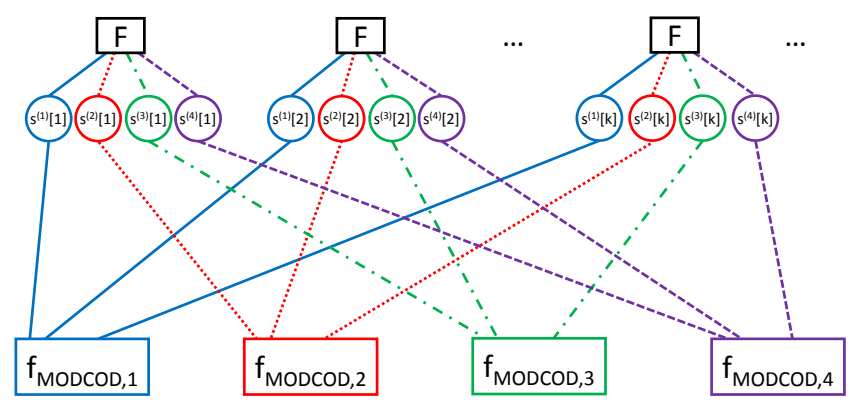

Figure 3. FG for joint detection of 4 bit streams in the case of symbol synchronous reception. Here, $s^{(1)}, s^{(2)}, s^{(3)}$ and $s^{(4)}$ denote $\tilde{x}_{1_{p}}, \tilde{x}_{2_{p}}$, $\tilde{x}_{1_{c}}$ and $\tilde{x}_{2_{c}}$, respectively.

A possible simplification consists in using a receiver with successive interference cancellation (SIC) that treats part of the interference as noise (IAN). Such a receiver runs through several stages $n(n=1,2, \ldots)$, whereby in each stage the previously decoded bit streams are cancelled from the observation, after which a novel subset of $N_{n}$ bit streams
Table I

SATELLITE SYSTEM PARAMETRES

\begin{tabular}{|l|l|}
\hline Satellite forward link & \\
\hline Diagram pattern & Provided by ESA [3] \\
\hline Number of beams & 245 \\
\hline Number of feeds & 245 \\
\hline Feed synchronization & Perfect synchronization \\
\hline Fading & No Fading \\
\hline Traffic distribution & Uniform \\
\hline Frequency band [GHz] & 20 \\
\hline Frequency coloring scheme & 2 \\
\hline Total Bandwidth [MHz] & 500 \\
\hline Beam bandwidth [MHz] & 250 \\
\hline
\end{tabular}

Table II

USER TERMINAL PARAMETRES

\begin{tabular}{|l|l|}
\hline User terminal & \\
\hline $\mathrm{Rx}$ antenna gain $[\mathrm{dB}]$ & 42.2 \\
\hline $\mathrm{Rx}$ noise temperature $[\mathrm{K}]$ & 235.34 \\
\hline Interference cancellation & Ideal cancellation \\
\hline
\end{tabular}

is decoded, while treating the signals corresponding to the remaining bit streams as noise. With this approach the FG employed in stage $n$ contains only $N_{n}$ (with $N_{n}<4$ ) MODCOD constraint nodes, which significantly reduces the complexity of the SPA. In stages with $N_{n}$ equal to 1 , only a single MODCOD decoder remains and iterative MUD is even no longer required.

The NCRS scheme uses the above approach to decode the conveyed information in 2 stages. In the first stage the messages $m_{1_{c}}$ and $m_{2_{c}}$ are jointly decoded, so $N_{1}=2$; the interference stemming from $m_{1_{p}}$ and $m_{2_{p}}$ is treated as noise (in Figure 3 only 2 MODCOD nodes remain). In the second stage, receiver 1 (receiver 2 ) decodes $m_{1_{p}}\left(m_{2_{p}}\right)$ treating $m_{2_{p}}\left(m_{1_{p}}\right)$ as noise; in both cases $N_{2}=1$ (in Figure 3 only 1 MODCOD node remains).

A possible approach to further simplify these schemes is to replace in the above the first stage, by 2 stages 1 and 1', respectively, with $N_{1}=N_{1^{\prime}}=1$. Then, for example, in stage 1 , the message $m_{1_{c}}$ is decoded, treating $m_{2_{c}}, m_{1_{p}}$ and $m_{2_{p}}$ as noise, while in stage 1' $m_{2_{c}}$ is decoded, treating $m_{1_{p}}$ and $m_{2_{p}}$ as noise. Of course this kind of simplification might result in a reduced performance.

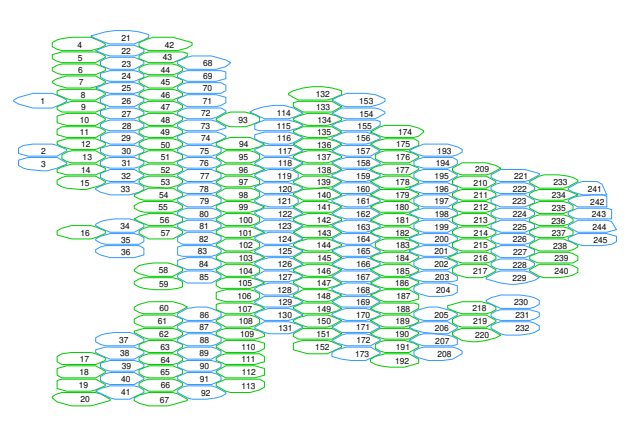

Figure 4. Beam pattern used for 2-colour NCRS. Each colour represents the use of half of the total spectrum. 


\section{NUMERICAL RESULTS}

The system level performance of NCRS has been tested for the scenario described in Table I. 50 Monte Carlo realizations have been run, with one user per beam randomly located at each realization. A benchmark 4-colour Frequency Division Multiplexing (FDM) is compared with 2-colour NCRS; the corresponding beam map is shown in Figure 4. For each realization, users are paired and rates are allocated following the description in Section V. Results are obtained for different transmission power values. Figure 5 shows the average total throughput with respect to the SNR at the center of the beams, which is given by $\mathrm{SNR}_{q}=\frac{\frac{P}{K}\left|h_{q q}\right|^{2}}{\sigma^{2}}$. It can be seen that the gain of 2-colour NCRS with respect to 4-colour FDM can be significant for lower SNR values (in the order of $20 \%$ ), and gets barely unnoticeable for higher SNR regimes. More specifically, Figure 6 depicts the relative gain, also for the case when the background interference is ideally suppressed, i.e., NCRS only deals with the interference within the pair of beams. Clearly, the presence of a significant background interference which cannot be addressed by NCRS, especially for higher SNR values, is drastically limiting the gain of this rate splitting technique.

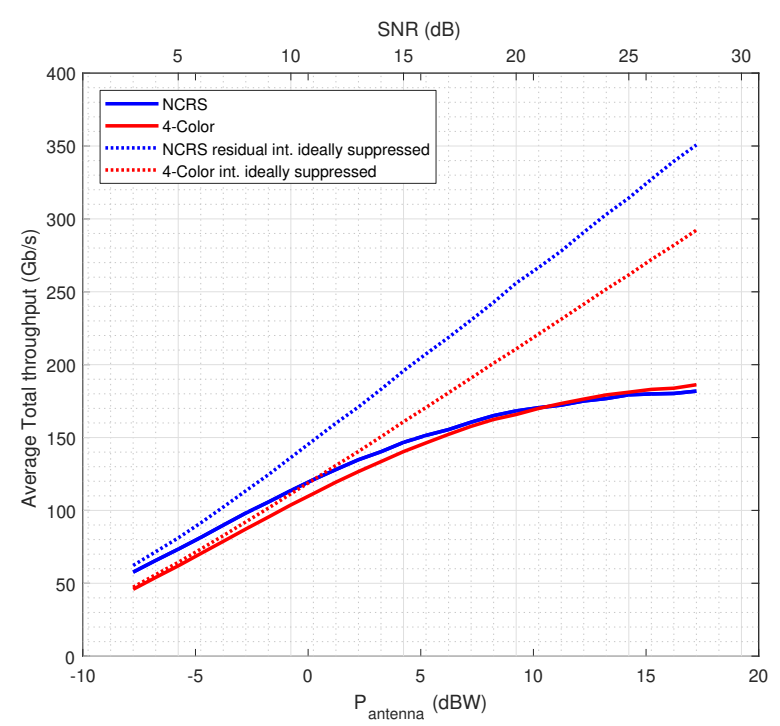

Figure 5. Comparison of overall maximum rate.

For a better understanding of the magnitude of the background interference in a 2-colour scheme, Figure 7 depicts the different levels of background interference when only the $k$ weakest interference signals are taken into account. The interference magnitude $I_{k}$ is obtained for the user within the $q$ th beam when that user is at the centre of the beam and $P_{\text {antenna }}=5.24 \mathrm{dBW}$, which corresponds to EIRP $=63 \mathrm{dBW}$. If a pair $(q, m)$ is made so that the $m$ th beam is the neighboring beam posing the highest interference magnitude, and this interfering signal is completely cancelled, then the corresponding carrier to interference ratio $(\mathrm{C} / \mathrm{I})$ is the one highlighted in red and it is given by $\mathrm{C} / \mathrm{I}=\frac{\frac{P}{K}\left|h_{q q}\right|^{2}}{I_{q m}}$. Although this result highly depends on the antenna radiation pattern, it clearly demonstrates a significant amount of background interference. Moreover, the SNR and the Signal-to-Interference-plus-Noise ratio (SINR) PDF for different locations of the user within the beam are shown in Figure 8, where $\operatorname{SINR}_{q}=\gamma_{q q}$. The impact of the background interference can be clearly seen in the degradation of the SINR curve. Simulation results, not shown here, show that a $20 \mathrm{~dB}$ reduction of this interference would be required for NCRS to achieve the performance without background interference in Figure 5. Even in such a case, the improvement of 2-colour NCRS with respect to 4-Colour FDM is limited to $20-25 \%$, as concluded from Figure 6. For further improvement, full frequency reuse could be considered. Unfortunately, the complexity increases significantly in that case, since three or more beams must be grouped, making group selection and rate optimization in NCRS significantly more involved.

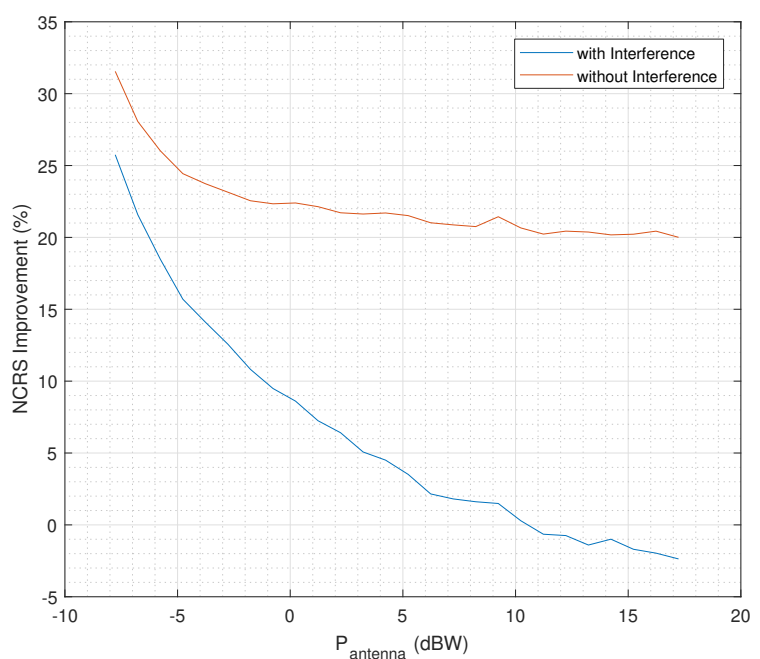

Figure 6. 2-colour NCRS gain with respect to 4-colour FDM. Percentage applies to the overall rate.

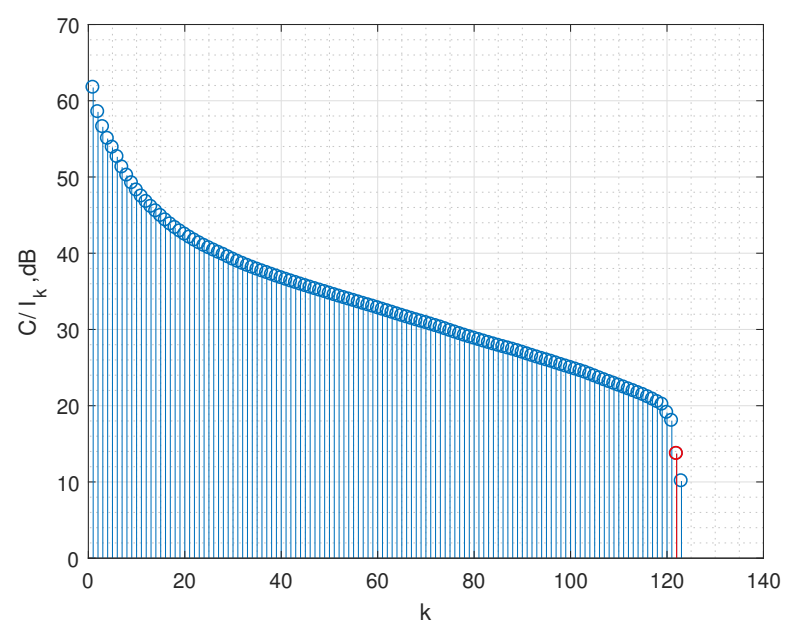

Figure 7. $C / I_{k}$, where $I_{k}$ is the sum of the $k$ weakest interfering signals. User allocated at the center of the beam. Pantenna $=5.24 \mathrm{dBW}$. 


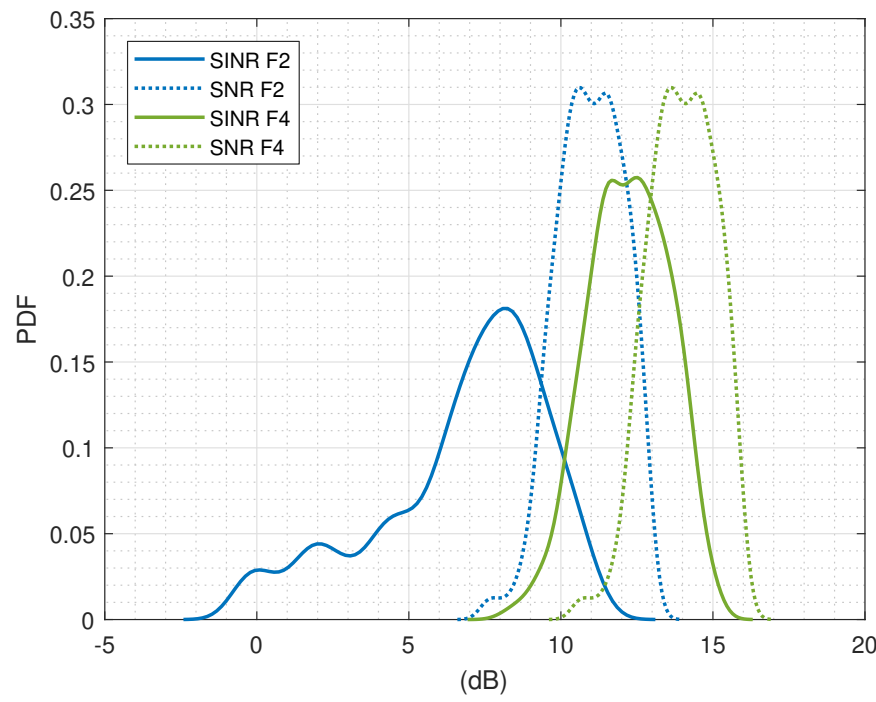

Figure 8. SNR and SINR pdf for $P_{\text {antenna }}=5.24 \mathrm{dBW}$.

\section{CONCLUSIONS}

Under the theoretical framework of MISO BC, interference enhancement is the way to achieve the GDoF in the MISO BC when CSIT is not available, relying on rate splitting to take advantage of the interference. Inspired by interference enhancement, Non-Coherent Rate Splitting has been presented as a scheme not requiring the channel phase information for multibeam scenarios with aggressive frequency-reuse. In order to limit the complexity, NCRS has been applied to pairs of users, following a heuristic approach to pair the corresponding beams. The parameters of the encoding were designed to maximize the sum-rate. With respect to the 4-colour FDM performance benchmark, NCRS obtains an improvement for low and moderate SNR values for the multibeam system under study. In particular, 20$25 \%$ improvement is achieved for low SNR, and 5-10\% for moderate SNR. For higher SNR, the performance of NCRS degrades due to the relevance of the additional untreated interfering sources with respect to the noise. Without the impairments of the background interference, NCRS would achieve an improvement around $25 \%$. Thus, the amount of background interference which cannot be treated by NCRS sets a bound on the achievable performance.

\section{ACKNOWLEDGMENT}

This work has been supported by European Space Agency funded activity SatNEx IV CoO2-Part 1 WI 3 "Non-Orthogonal Superposition Techniques for Multi-Beam Satellite Networks". The views of the authors of this paper do not reflect the views of ESA. This work was partially funded by the Agencia Estatal de Investigacion (Spain) and the European Regional Development Fund (ERDF) through the projects TERESA- TEC2017-90093-C31-R (AEI/FEDER,UE) and MYRADA (TEC2016-75103-C2-2-R). Funded also by the Catalan Government under project 2017-SGR1479, and Galician Government under project ED431C 2017/53.

\section{REFERENCES}

[1] B. Devillers, A. Perez-Neira, and C. Mosquera, "Joint linear precoding and beamforming for the forward link of multi-beam broadband satellite systems," in IEEE Global Telecommunications Conference GLOBECOM 2011, Houston,TX,USA, Dec 2011, pp. 1-6.

[2] G. Taricco, "Linear precoding methods for multi-beam broadband satellite systems," in European Wireless 2014; 20th European Wireless Conference, Barcelona, Spain, May 2014, pp. 1-6.

[3] M. A. Vazquez, A. Perez-Neira, D. Christopoulos, S. Chatzinotas, B. Ottersten, P. D. Arapoglou, A. Ginesi, and G. Tarocco, "Precoding in Multibeam Satellite Communications: Present and Future Challenges," IEEE Wireless Communications, vol. 23, no. 6, pp. 88-95, December 2016.

[4] M. Caus, A. I. Perez-Neira, M. Angelone, and A. Ginesi, "An innovative interference mitigation approach for high throughput satellite systems," in 2015 IEEE 16th International Workshop on Signal Processing Advances in Wireless Communications (SPAWC), Stockholm, Sweden, June 2015, pp. 515-519.

[5] N. Alagha and A. Modenini, "On capacity measures for multi-beam satellite systems analyses," in 8th Advanced Satellite Multimedia Systems Conference - 14th Signal Processing for Sapce Communications Workshop, Palma de Mallorca, Spain, Sep. 2016.

[6] G. Colavolpe, A. Modenini, A. Piemontese, and A. Ugolini, "Multiuser detection in multibeam satellite systems: Theoretical analysis and practical schemes," vol. 65, no. 2, pp. 945-955, Feb 2017.

[7] R. D. Gaudenzi, N. Alagha, M. Angelone, and G. Gallinaro, "Exploiting code division multiplexing with decentralized multiuser detection in the satellite multibeam forward link," International Journal of Satellite Communications and Networking, Aug. 2017.

[8] A. Ugolini, G. Colavolpe, and A. Vanelli-Coralli, "A system level approach to the application of multiuser detection in multibeam satellite systems," in 2017 International Symposium on Wireless Communication Systems (ISWCS), Bologna, Italy, Aug 2017, pp. 66-71.

[9] M. Caus, A. Pastore, M. Navarro, T. Ramírez, C. Mosquera, N. Noels, N. Alagha, and A. I. Perez-Neira, "Exploratory Analysis of Superposition Coding and Rate-Splitting for Multibeam Satellite Systems," in 15th International Symposium on Wireless Communications Systems, Lisbon, Portugal, 2018.

[10] S. A. Jafar et al., "Interference alignment-a new look at signal

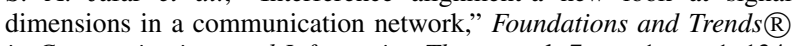
in Communications and Information Theory, vol. 7, no. 1, pp. 1-134, 2011.

[11] T. Gou and S. A. Jafar, "Optimal Use of Current and Outdated Channel State Information: Degrees of Freedom of the MISO BC with Mixed CSIT," IEEE Communications Letters, vol. 16, no. 7, pp. 1084-1087, July 2012.

[12] H. Joudeh and B. Clerckx, "Sum-Rate Maximization for Linearly Precoded Downlink Multiuser MISO Systems With Partial CSIT: A Rate-Splitting Approach," IEEE Transactions on Communications, vol. 64, no. 11, pp. 4847-4861, Nov 2016.

[13] A. G. Davoodi and S. A. Jafar, "GDoF of the MISO BC: Bridging the gap between finite precision CSIT and perfect CSIT," July 2016, pp. 1297-1301.

[14] T. Han and K. Kobayashi, "A new achievable rate region for the interference channel," vol. 27, no. 1, pp. 49-60, Jan 1981.

[15] R. H. Etkin, D. N. C. Tse, and H. Wang, "Gaussian interference channel capacity to within one bit," vol. 54, no. 12, pp. 5534-5562, Dec 2008.

[16] A. G. Davoodi and S. A. Jafar, "Transmitter Cooperation Under Finite Precision CSIT: A GDoF Perspective," IEEE Transactions on Information Theory, vol. 63, no. 9, pp. 6020-6030, Sept 2017.

[17] J. Nocedal and S. J. Wright, Numerical Optimization, 2nd ed. New York: Springer, 2006.

[18] F. R. Kschischang, B. J. Frey, and H. A. Loeliger, "Factor graphs and the sum-product algorithm," IEEE Transactions on Information Theory, vol. 47, no. 2, pp. 498-519, Feb 2001. 